\title{
Path Loss Modeling of WLAN and WiMAX Systems
}

\author{
Imran Israr*, Mustafa Shakir*, Mahmood Ashraf Khan*, Shahzad A. Malik*, Shahid A. Khan* \\ * Department of Electrical Engineering, COMSATS Institute of Information Technology, \\ Islamabad
}

\begin{tabular}{l}
\hline \hline Article Info \\
\hline Article history: \\
Received Feb 7, 2015 \\
Revised May 28, 2015 \\
Accepted Jun 14, 2015 \\
\hline
\end{tabular}

Keyword:

Models

Pathloss

Scheduling

WiMAX

WLAN

\begin{abstract}
With the advancement in technology, there was need for efficient and high speed internet through which we could have access to multiple networks as per the user requirement. WLAN met this need to some extent but, due to its low range it was not recommended commercially. With the introduction of WiMAX there was an emerging need to select the best network amongst WiMAX or WLAN depending upon the user location. Path loss with respect to these particular networks also needs to be compared. In this paper we compare the path lossmodeling for WiMAX and WLAN systems. Different Models have been compared with each other to know which model performs better by keeping same simulation environment. Path Loss models used for WLAN are Okumura, Hata, Cost-231 and Free Space Path Loss whereas models used for WiMAX are Free Space Path Loss, Okumura-Hata, Cost231-Hata and Stanford University Interim. In case of WiMAX three different scenarios Urban, Sub-Urban and Rural is considered where as in case of WLAN only outdoor environment is considered. With the Path Loss comparison, power received for these two technologies; WiMAX, and WLAN are also simulated. MATLAB is the tool used for simulations. Antenna Specifications for WiMAX and WLAN is kept same for all simulation environments.
\end{abstract}

Copyright (C) 2015 Institute of Advanced Engineering and Science. All rights reserved.

\section{Corresponding Author:}

Imran Israr,

Department of Electrical Engineering,

COMSATS Institute of Information Technology,

Islamabad

\section{INTRODUCTION}

In radio propagation spatial, channels and temporal variations are usually calculated using three main scales. Signal variation on small scale called as fast fading, signal variation on small area average known as shadowing and variations over large area distance known as path loss. Path loss plays an important role in determining distance between transmitter and receiver in mobile systems and also it is a good assessment perimeter to know how radio propagation signal varies with distance. This paper is on calculating path loss of Wireless Local Area Network (WLAN) and Worldwide Interoperability for Microwave Access (WiMAX) by using path loss models which are previously designed by researchers for both of these techniques.

\section{A. WiMAX}

IEEE 802.16 is well known as WiMAX. It supports high radius and high data rates up to several $\mathrm{km}$ and Mbit/s respectively. It has three types of topologies Point to Point (P2P), Point to Multipoint (P2MP) and Multipoint to multipoint (MP2MP) or (Mesh). 


\section{B. WLAN}

WLAN links one or more devices using some wireless distribution method. The access point is a part of wider internet through WLAN. With this technique one can move around within a local area network thus utilizing the services of internet. Wireless network occupies an important function in field of LANs. ALOHA was the first computing developed, later with time different protocols has been developed. It is found that wireless LANs are an adjunct to traditional wired LANs, to overcome the problem of mobility, locations where wired structure is very difficult and due to limitations of coverage area. Wired and wireless are the two basic types of computing networks. Our work addresses mainly wireless local network (WLAN).

\section{RELATED WORK AND MOTIVATION}

WiMAX system evaluation methodology is explained in [1] by authors. System simulation modeling for center cell approach, sector assessment, power control and application traffic models have also been explained in this book. MAC layer and PHY layer modeling have also been done. Our related work is on channel models which include path loss, shadowing, multipath and empirical path loss models which have been discussed in [1].

Empirical PL models have been used to estimate the PL for C-Band Airport Surface communication is discussed by authors in [2].

Different NLOS and LOS paths are used to calculate the PL in different areas of airport. In this paper authors have used different measurements which are collected from LOS and NLOS paths to develop path loss parameters and path loss models for different zones for large airports. Antenna characteristics which are used are, gain of base station is $15 \mathrm{dbi}$ and mobile station antenna gain of 6dbi. Beam width in azimuth is 120 degrees while it is only 6 degrees in elevation. Different scenarios have been supposed to collect data for establishing the path loss model.

In [3] authors have evaluated Path Loss models at WiMAX cell-edge. WiMAX coverage area is one of the most important factors that need consideration while developing a system. Predicting and finding PL is very important in planning and designing mobile systems. In this paper author presented comprehensive study on path loss models which are developed to provide high throughput, low PL and wide coverage for mobile WiMAX. Authors calculated path loss, compared the path loss values, through path loss they calculated outage probability and also determined link budget. WiMAX frequency of $3.5 \mathrm{GHz}$ is used with range variation from $1 \mathrm{~km}$ to $50 \mathrm{~km}$.

Comparison of models of WiMAX in rural environments at $3.5 \mathrm{GHz}$ is discussed by authors in [4]. Models which are used for comparison are Cost-Hata, Erceg, Dual-Slope, SUI, Macro model and ECC-33. This comparison is for mobile as well as fixed wireless systems like WiMAX.

Authors in [13] proposed a combined spatial multiplexing MIMO scheme with beamforming for high data rate wireless communication. The proposed transmission scheme combines the benefits of both techniques and the system was able to transmit parallel data streams aswell as provide beamforming gain. The proposed hybrid technique improved the system spectral efficiency performance significantly than the conventional MIMO, spatial multiplexing and beamforming techniques when used alone under the same simulation environment

Authors in [14] evaluated the performance of Multiple Input Multiple Output (MIMO) channels in fading environment, Both cases of correlated and uncorrelated MIMO channels are considered under the condition when Channel State Information (CSI) is not known at transmitter and CSI is known at receiver side, Authors have compared the capacity of 2x2, 3x3 and 4x4 MIMO channels and have shown that capacity increases linearly with increase in the number of antennas at transmitter and receiver side

Authors in [15] provide a survey of the basic mechanisms which influence the propagation of electromagnetic waves in hilly areas. Three empirical models: COST231-Hata, Okumura-Hata and Egli which are suitable for path loss prediction for such area are presented. By using these propagation models the broadcast signal strength are predicted for this type of environment. Measurement results of signal strength in UHF band obtained in Idanre Town of Ondo State Nigeria are presented and compared with the results predicted by using the propagation models moreover authors have also proposed A modified COST231-Hata radio wave propagation model was developed and implemented with Matlab GUI (Graphical User Interface) for simulation.

\section{PATH LOSS MODELS OF WIMAX AND WLAN}

Path loss measures the signal loss due to different climatic effects. It tells about decrease in signal power due to different climate affects, environmental and geographical conditions. Through Path Loss we know about radio signals propagation in different environments. Link budget usually uses PL to predict 
received power at the output. There are many models that are designed to predict PL of different techniques operating in different frequency bands. These models are used to predict PL almost exactly. Using these models variation of radio signals and path loss they experience while traveling through different environments of urban, sub-urban and rural areas are predicted. Path Loss is the main problem while dealing with wireless signals. Due to Path Losses signals do not reach receiver accurately and hence information is lost in between. Due to Path Losses drop in signal power occurs hence proper internet services is not served.

\section{A. WiMAX}

In this paper we have analyzed four different models which have been proposed by the researchers in past at operating frequency of $3.5 \mathrm{GHz}[11,12,13-16]$. These models have been especially proposed for WiMAX so that they are able to be operated at frequency of 3.5GHz. In our thesis i have considered Free Space Path Loss model (FSPL) as reference model. This model is used as idealistic model. We take it as reference model so, we can compare our other models with reference to this model.

\section{B. WLAN}

In this section PL models of WLAN will be explained. 4 different models are used for calculation of WLAN at frequency of $2.4 \mathrm{GHz}$ and considering three type of environments Urban, Sub-Urban and Rural. PL models which are used for prediction of PL in all three environments are FSPL, Okumura, Hata and Cost231. Original models are used with correction factors so that correct path loss can be predicted at frequency of $2.4 \mathrm{GHz}$.

\section{SIMULATION AND RESULTS}

Simulations and results have been discussed in this section. Simulation parameters are given in table III. Matlab is the tool that is used for simulation. For comparison of different models of WiMAX and WLAN simulation parameters are kept same, so that comparison can be done by keeping everything same.

Table 3. Simulation Parameters

\begin{tabular}{cc}
\hline Parameters & Values \\
\hline Base Antenna Transmission Power (pt) & $50 \mathrm{db}$ \\
Transmission Antenna Height (ht) & $30 \mathrm{~m}$ \\
Receiving Antenna Height (hr) & $10 \mathrm{~m}$ \\
Operating Frequency WiMAX & $3.5 \mathrm{GHz}$ \\
Operating Frequency WLAN & $2.4 \mathrm{GHz}$ \\
Distance TX-RX WiMAX & $5 \mathrm{~km}$ \\
Transmission Antenna Gain (Gt) & $15 \mathrm{dbi}$ \\
Receiving Antenna Gain (Gr) & $15 \mathrm{dbi}$ \\
Distance TX-RX WLAN & $300 \mathrm{~m}$ \\
\hline
\end{tabular}

Table 3 shows simulation parameters with their values, these parameters kept constant for every simulation so that a proper comparison can be done between different models of WiMAX and WLAN.

\section{A. Path Loss of WiMAX at $3.5 \mathrm{GHz}$}

Free Space Path Loss (FSPL) is shown in figure 5. Path Loss (PL) is plotted using range of distance up to $5 \mathrm{~km}$. PL is increasing with increase in distance from $0 \mathrm{~km}$ to $5 \mathrm{~km}$. Increase in path loss is due to climate affects, reflection from buildings, geographic issues. At 0km FSPL have PL of $60 \mathrm{db}$, at $1 \mathrm{~km}$ distance it has PL of $100 \mathrm{db}$. At maximum distance of 5km it has PL of 114db value. 


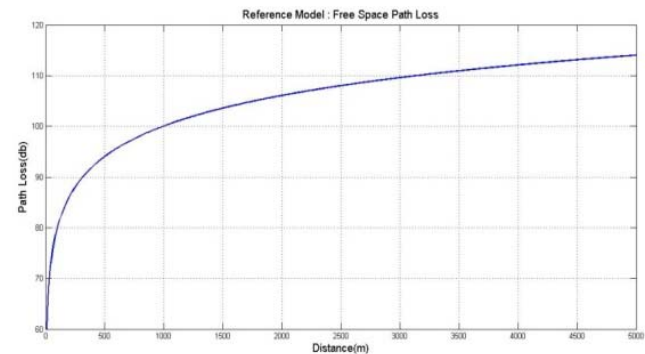

Figure 5. Free Space Path Loss
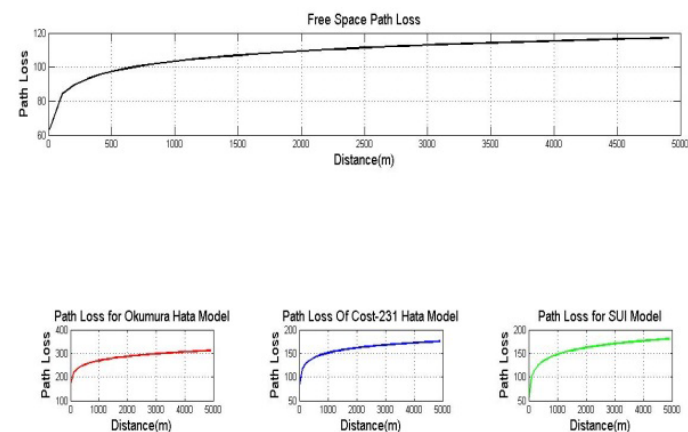

Figure 6. Path Loss in Urban Area of WiMAX models with Reference Model

Comparisons of different Models Okumura-Hata, Cost231-Hata and Sui, with keeping FSPL as reference model using different environment Urban, Sub-Urban and Rural are given in figures 6, 7, 8 respectively.

Comparison of different Models Okumura-Hata, Cost231-Hata and Sui, with keeping FSPL as reference model using different environment Urban, Sub-Urban and Rural are shown in figure 6.PL of different models of WiMAX in Urban Area is shown in figure 6. Models which are used for WiMAX are Okumura-Hata, Cost231-HATA and SUI model. FSPL is used as reference model and rest of models are compared with it. FSPL has lowest PL, with SUI being second with lowest Path Loss, Cost231-Hata have PL almost equal to that of SUI model. Highest PL in this scenario is of Okumura-Hata model.
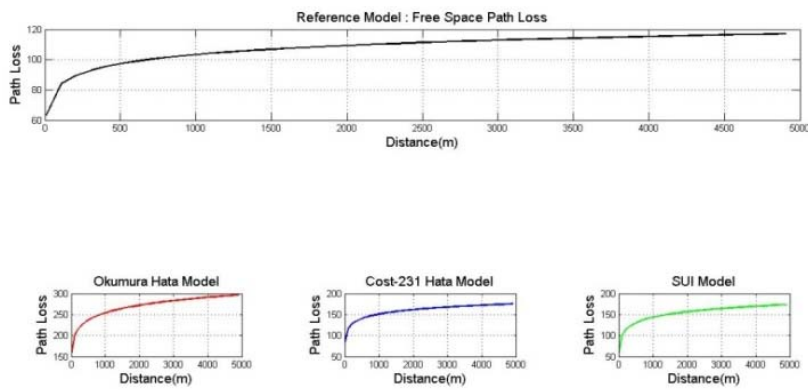

Figure 7. Path Loss in Sub-Urban Area of WiMAX models with Reference Model

Figure 7 shows Path Loss of different Models of WiMAX in sub-urban area. FSPL is used as reference Model for comparing of different models with it. This figure has same characteristics as PL in urban area. SUI has lowest PL among Okumura-Hata and Cost231-Hata. Cost231-Hata is close to SUI. 

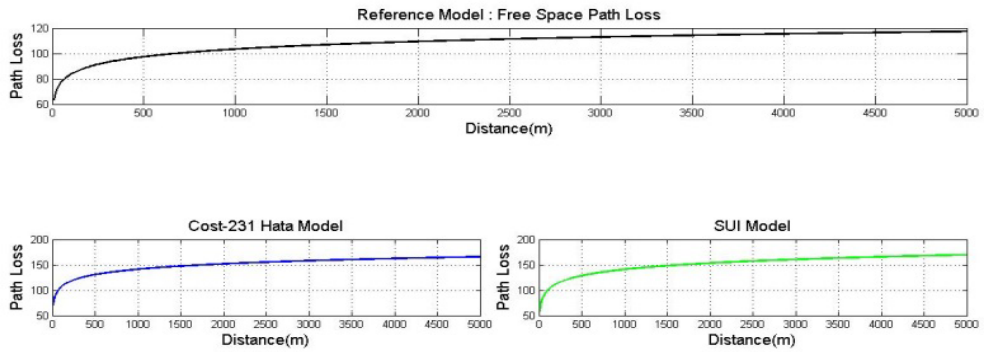

Figure 8. Path Loss in Rural Area of WiMAX models with Reference Mode

Figure 8 shows PL or WiMAX in rural area. Okumura does not have any model designed for calculation of PL in rural area in this case. Both Cost231-Hata model and SUI are compared by keeping FSPL as reference model. Sui has lowest PL then Cost231-Hata.

Path Loss comparison of each model with itself, in Rural, Sub-Urban and Urban area is shown in figures 9, 10, 11 and given below. These graphs give an idea of each model performance in different areas.

Figure 9 shows Okumura-Hata Path Loss model of WiMAX in urban and sub-urban areas. It has more PL in Urban area then in sub-urban. Reason is that in urban area there are more obstacles between signal paths than sub-urban. Other reason is effect of climate, which affects signal during propagation.

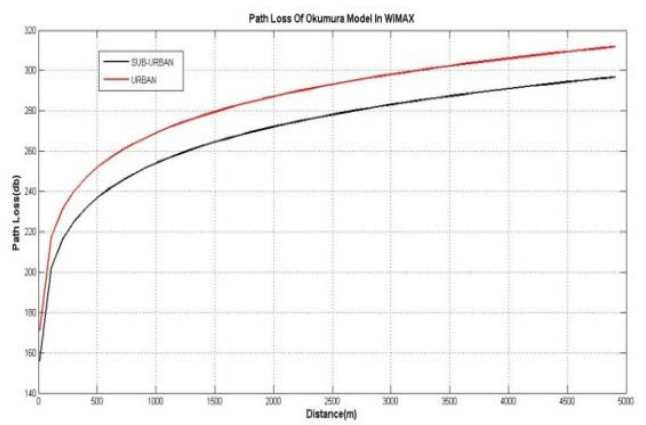

Figure 9. Path Loss of Okumura-Hata Model of WiMAX

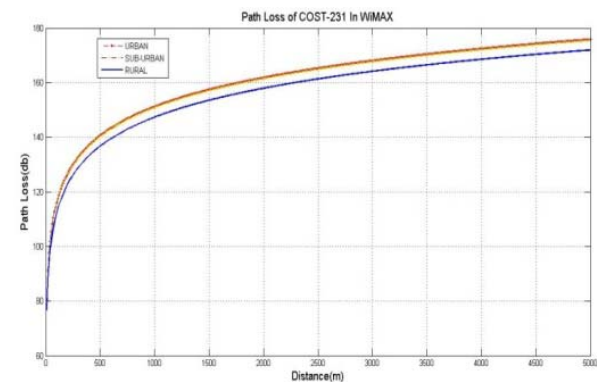

Figure 10. Path Loss of Cost231-Hata Model of WiMAX

Performance with respect to PL of Cost231-Hata in three different environments is shown in Figure 10. This figure shows that urban has highest PL then sub-urban and rural but both urban and sub-urban are very close to each other and almost nearly equal. 


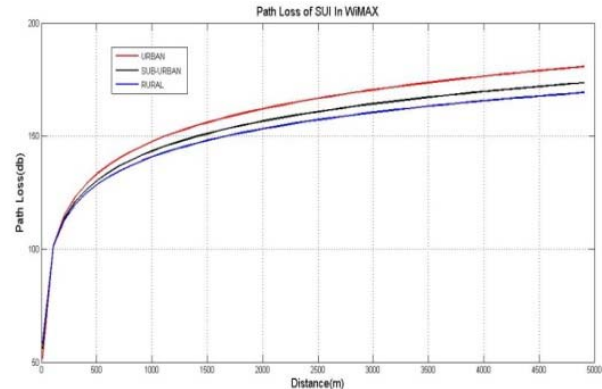

Figure 11. Path Loss of SUI Model of WiMAX

PL comparisons of SUI model in three different scenarios or environments are shown in Figure 11. SUI has more PL in urban area compared to sub-urban and rural area. Reason is same due to more obstacles and environment factors. SUI model used is upgraded for WiMAX to be operated on frequency of $3.5 \mathrm{GHz}$.

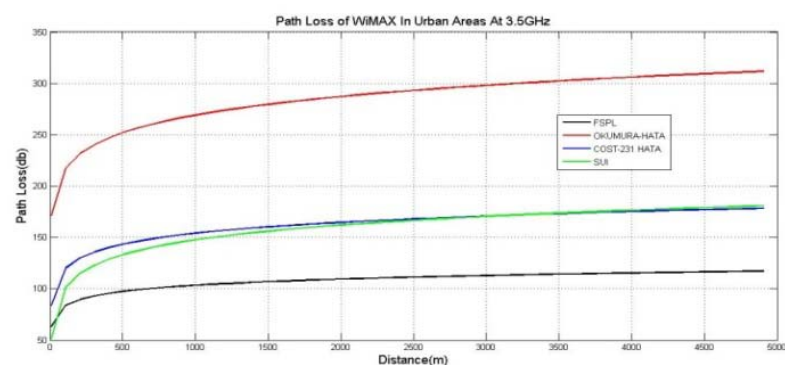

Figure 12. Comparison of Path Loss in Urban Area of WiMAX

Figure 12 shows comparison of different models in urban environment. Okumura-Hata Model has highest PL after Okumura-Hata, Cost231-Hata has higher PL then SUI and model with least PL is FSPL.

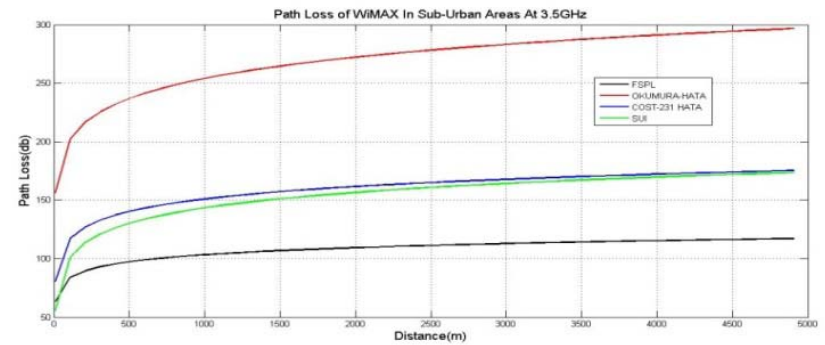

Figure 13. Comparison of Path Loss in Sub-Urban Area of WiMAX

In Figure 13 comparison of PL models are shown in sub-urban environment. FSPL has lowest PL then SUI and Cost231-Hata are close to each other. Okumura has highest PL. 


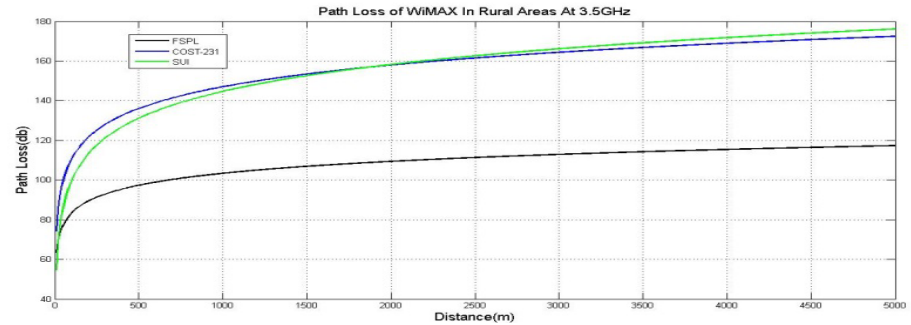

Figure 14. Comparison of Path Loss in Rural Area of WiMAX

Comparison of PL in rural environments among different models are shown in Figure 14. OkumuraHata model does not exist for rural area. Cost231-Hata and SUI are close to each other. In this graph Cost231 up to 2.5k has higher PL, but SUI has more PL after 2.5k. Both Cost231-Hata and SUI have same values of PL. at distance of $1850 \mathrm{~m}$.

\section{B. Path Loss of WLAN at $2.4 \mathrm{GHz}$}

Path Loss of models of WLAN in outdoor environment is shown in figure 15. Okumura, Hata and Cost-231 are compared with FSPL which is used as a reference model for comparison by me. Okumura have less PL then Hata model and Cost-231. Cost-231 has highest PL compared to other models.

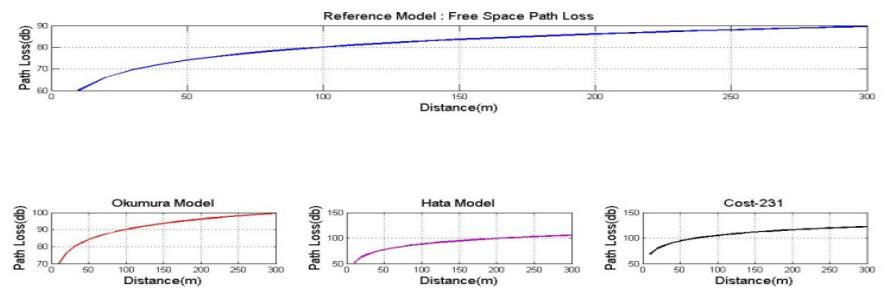

Figure 15. Path Loss Comparison in Outdoor Environment of WLAN models With Reference Model

Figure 16 shows PL comparison of WLAN models in Outdoor area. In this area all models have less difference between them other than Cost-231. Up to 150 meters Hata lags Okumura model but after $150 \mathrm{~m}$ Hata leads Okumura model as shown in figure.

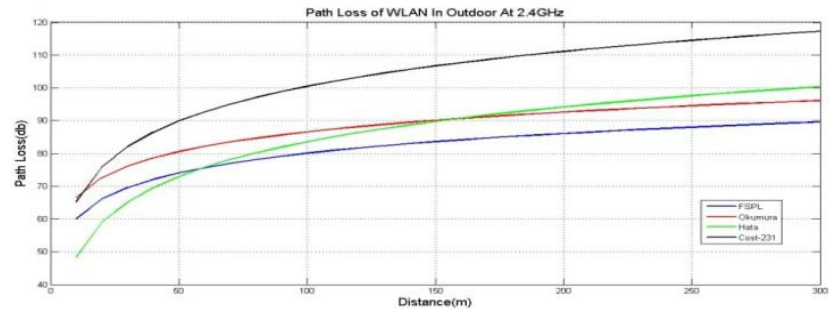

Figure 16. Path Loss Comparison of WLAN Models in Outdoor Environment.

\section{CONCLUSION AND FUTURE WORK}

In this paper path loss models of WLAN and WiMAX have been compared. Existing models are amended to be able to work for WiMAX at frequency of 3.5GHz. Models which are used for comparison in WLAN are Okumura, Hata, FSPL and Cost-231 and models which are used for comparison in WiMAX are FSPL, Cost231-Hata, Okumura-Hata and SUI.

We have taken only two important technologies WLAN and WiMAX and performed path loss modeling to know that, how path loss also affects them. Three different scenarios have been used for 
comparison in case of WiMAX, each scenario has its own properties and structures. Urban area considered having high building and large obstacles between signal paths, there are very few plain areas. Sub-Urban area is considered as an area with intermediate height of building and also less obstacles in signal path as compared to Urban and more plain lands. Rural is considered as flat terrain with very few buildings and large plain. For WLAN only outdoor environment is considered because WLAN is not affected by environments like urban, rural and sub-urban.

Aim of our work was to show that, how PL affects two technologies and how path loss varies in different environments. Due to multipath affect and NLOS environments in urban area, due to high buildings and populated areas, Path Loss in Urban area is very high as compared to Sub-urban and rural. Sub-urban includes less high buildings due to which its PL is high as compared to area where there are more plain areas means i.e. in rural area.

Our future work includes developing a path loss model which is more feasible and can give more accurate results. I want to develop a model based on values recorded in the region of Pakistan and which gives an idea of how signal varies in power in Pakistan and how to develop a better network by considering PL analysis

\section{REFERENCES}

[1] E.H. Jan, R. Vidar, A.M. Tor, "Empirical Path Loss Models for C-Band Airport Surface Communications ", IEEE transaction on antenna and propagation, Vol. 60, July, 2012

[2] A. Mohammad, A. Tughtul, T. John, E. Ahmot, "Evaluation of Path Loss models at WiMAX cell-edge", 978-14244-8704-2/11/26.00 @2011 IEEE.

[3] K. Vivek, D.K. Gupta, B. Nidhika, "Comparison of Path Loss Models For WiMAX in Rural Environment at 3.5 HGz." International Journal of Engineering Science and Technology (IJEST), Vol. 3, No. 2, Feb 2011.

[4] H. MN, S. Robert, B. Eduardo, A. John, "Deployment and Performance Issues of an Integrated Wireless Sensor Network and Wireless Mesh Campus Network", CSNDSP, 2010

[5] R. Merdeni, T.S. Priya, "Optimised COST-231 Hata Models for WiMAX Path Loss Prediction in Suburban and Open Urban Environments", Modern Applied Sciences, Vol. 4, No. 9, September 2010.

[6] Wei Song, "Heterogeneous Multi Hop Transmission of Compressed ECG Data from Wireless Body Area Network", 978-1-61284-233-2, 2011

[7] Akkasli, Cem. "Methods for Path loss Prediction." (2009).

[8] V.S. Abhayawardhana, I.J. Wassel, D. Crosby, M.P. Sellers, M.G. Brown, "Comparison of empirical propagation path loss models for fixed wireless access systems", 61th IEEE Technology Conference, Stockholm, pp. 7377, 2005.

[9] Josip Milanovic, Rimac-Drlje S, Bejuk K, "Comparison of propagation model accuracy for WiMAX on 3.5GHz", 14th IEEE International conference on electronic circuits and systems, Morocco, pp. 111-114. 2007.

[10] S. Ullah, K.S. Kwak, "An Ultra Low-power and Traffic-adaptive Medium Access Control Protocol for Wireless Body Area Network", J Med Syst (2012) 36:1021-1030 DOI 10.1007/s10916-010-9564-2.

[11] E.H. Jan, R. Vidar, A. M. Tor, "Empirical Path Loss Models for C-Band Airport Surface Communications", IEEE transaction on antenna and propagation, Vol. 60, July, 2012

[12] Simic I. lgor, Stanic I., and Zrnic B., "Minimax LS Algorithm for Automatic Propagation The Model Tuning", Proceeding of the 9 Telecommunications Forum (TELFOR 2001), Belgrade, Nov.2001.

[13] O. Kehinde. O. Erastus, "Capacity Enchancement for High Data Rate Wireless Communication System", International Journal of Electrical and Computer Engineering (IJECE), Vol. 4, No. 5, October 2014, pp. 800 809.

[14] M. M. Lodro, M. H. Abro, "Ergodic Capacity of MIMO Correlated Channels in MultipathFading Environment with known Channel State Information”, International Journal of Electrical and Computer Engineering (IJECE), Vol.2, No.5, October 2012, pp. 691 697.

[15] F. J. Oluwole, O. Y. Olajide, "Radio Frequency Propagation Mechanisms and Empirical Models for Hilly Areas", International Journal of Electrical and Computer Engineering (IJECE), Vol. 3, No. 3, June 2013, pp. 372 376.

\section{BIOGRAPHIES OF AUTHORS}

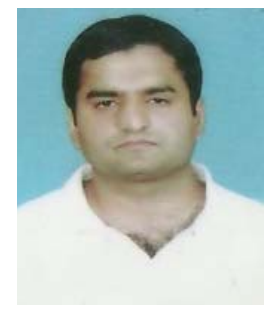

Imran Israr got his Master Degree in Electrical Engineering Specialization in Telecommunication Engineering from Comsats Institute of Information and Technology Islamabad. He is Network Engineer (NOC and CORE) at QUBEE ISP Islamabad. He has worked for Pakistan Consortium for Advance Research in Electrical Engineering and carried out research in Wireless Communication and Techniques. His areas of Interest include Wireless Networks, Wireless Sensor Networks, Communication Systems, Routing and Switching. 


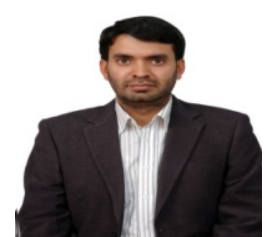

Dr. Mustafa Shakir got his $\mathrm{PhD}$ in Information and Communication Engineering from Beijing University of Posts and Telecommunications, China. He is an Assistant Professor at Department of Electrical Engineering, COMSATS Institute of Information Technology, Islamabad. He has worked for Pakistan Telecommunications Company Limited and conducted sessions and trainings on Broadband Access, Next Generation Networks, IPTV, Data Communications and Understanding Telecom Networks and Services. His areas of interest include wireless networks and communication systems, ad hoc networks, sensor networks and next generation networks.

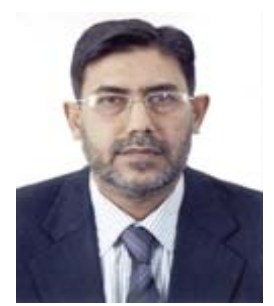

Dr. Mahmood Ashraf Khan got his PhD from the University of Aston in Birmingham, UK. $\mathrm{He}$ is serving as Director, Centre for Advanced Studies in Telecommunications (CAST) at COMSATS Institute of Information Technology, Islamabad. He has over seventeen years of services at Pakistan Telecommunication Company Ltd, Islamabad, in different capacities. Dr. Mahmood has an extensive experience in conducting research \& development programs at national and international level and providing consultancy services. He has coordinated with different international funding organizations and honored with excellence award from the Ministry of Science and Technology, Government of Pakistan. His research interests include data communication systems, transport and access related networks and technologies.

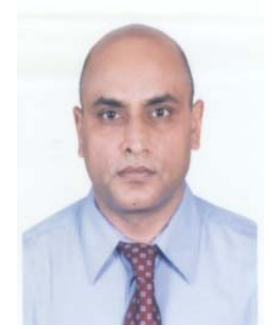

Dr. Shahzad A. Malik is a Professor at the Department of Electrical Engineering, COMSATS Institute of Information Technology, Islamabad. He holds a B.Sc. in Electrical Engineering from University of Engineering and Technology (UET), Lahore, Pakistan and M.S. and Ph.D. in Telecommunications from ENST, Paris, France. His research interests include wireless and mobile networks, integration of heterogeneous wireless networks, MAC and transport layer protocols for wireless networks, QoS and performance analysis of networks. He has published papers in several areas including radio resource allocation, mobility management, performance and capacity evaluation and quality of service provisioning in wireless networks. He has taught courses on wireless communication, mobile networks, computer networks, digital communications and performance analysis of computer networks.

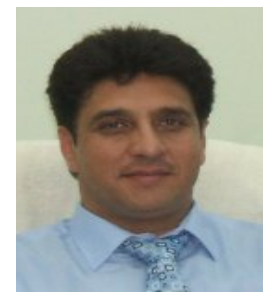

Prof. Dr. Shahid A. Khan is serving as Dean, Faculty of Engineering/ In charge Academics at COMSATS Institute of Information Technology, Islamabad. $\mathrm{He}$ did his $\mathrm{PhD}$ in Telecommunication Engineering and MS in Electronic Engineering from UK. He has about 24 years of experience in research, teaching and working in IT industries. His research publications encompass several papers published in international journals/conferences. On the basis of his meritorious services he has been honored with multiple awards and memberships in the field of engineering. He has played pivotal role in establishment of academic collaboration between CIIT and many international universities, especially with Lancaster University UK, QMUL UK, The University of Sheffield UK and NDSU U.S.A. 